\title{
ON SEMI INNER PRODUCT SPACES OVER QUATERNIONS
}

\author{
DILIP KUMAR SEN \\ (Received 19 June 1980; Revised 20 November 1980, and 3 September 1982) \\ Communicated by W. D. Wallis
}

\begin{abstract}
In this note semi inner product spaces over quaternions are studied. We investigate the properties of complex strict convexity over $C$, left and right $Q$-complex strict convexity over $Q$, and left and right quaternion strict convexity. Finally we discuss the permanence properties with respect to formation of products.
\end{abstract}

1980 Mathematics subject classification (Amer. Math. Soc.): $46 \mathrm{C} 10$.

Keywords and phrases: left and right semi inner products, $Q$-complex, $Q$-strict convexity.

\section{Introduction}

Lumer (1961) introduced the concept of semi inner product spaces over $K$ ( $K=R$ or $C$ ). Generalising this concept Torgashev (1974) introduced the concept of semi inner product spaces over quaternions and studied some of its properties. In this note, we obtain some further results on semi inner product spaces over quaternions.

Throp and Whitley (1967) introduced the concept of complex extreme points for studying the Banach space valued analytic functions. Later on, Istratescu (1979a, 1979b) studied complex extreme points and complex strictly convex spaces in detail. Here we give a sufficient condition for complex strict convexity over $C$ or $Q$. We also introduce and study left and right quaternion strictly convex spaces.

(c) 1984 Australian Mathematical Society $0263-6115 / 84 \$ A 2.00+0.00$ 
In particular characterizations of left and right quaternion strict convexity of product space with $l_{p}$-norm are given. Here vector spaces over quaternions and normed spaces over quaternions are all in the sense of Torgashev (1974).

\section{Left and right semi inner product spaces}

We recalll the following from Torgashev (1974).

Definition 2.1. Let $X$ be a vector space over $Q$. Then a s.i.p on $X$ is a functional ( , ): $X \times X \rightarrow Q$ satisfying the following properties:

(a) $\left(x+x^{\prime}, y\right)=(x, y)+\left(x^{\prime}, y\right)$,

$\left(\mathrm{b}^{\prime}\right)(\lambda x, y)=\lambda(x, y)$

$\left(\mathrm{b}^{\prime \prime}\right)(x, \lambda y)=(x, y) \bar{\lambda}$,

$\left(\mathrm{b}^{\prime \prime \prime}\right)(x \lambda, y)=(x, y \bar{\lambda})$,

(c) $(x, x) \geqslant 0,=0$ only if $x=0$,

(d) $|(x, y)|^{2} \leqslant(x, x) \cdot(y, y)$

for each $x, x^{\prime}, y \in X$ and each $\lambda \in Q$.

Note 2.2. We shall call a functional (, ) as defined above a left s.i.p. A vector space $X$ over $Q$ with a left s.i.p. will be called a left s.i.p. space over $Q$.

We now introduce the notion of right s.i.p. in the following way.

DefinITION 2.3. A right s.i.p. on a vector space $X$ over $Q$ is a functional $\langle$,$\rangle :$ $X \times X \rightarrow Q$ satisfying the following properties:

(a) $\left\langle x+x^{\prime}, y\right\rangle=\langle x, y\rangle+\left\langle x^{\prime}, y\right\rangle$,

(b') $\langle x \lambda, y\rangle=\langle x, y\rangle \lambda$,

$\left(\mathrm{b}^{\prime \prime}\right)\langle x, y \lambda\rangle=\bar{\lambda}\langle x, y\rangle$,

$\left(\mathrm{b}^{\prime \prime \prime}\right)\langle\lambda x, y\rangle=\langle x, \bar{\lambda} y\rangle$,

(c) $\langle x, x\rangle \geqslant 0$, $=0$ only if $x=0$,

(d) $|\langle x, y\rangle|^{2} \leqslant\langle x, x\rangle \cdot\langle y, y\rangle$

for each $x, x^{\prime}, y \in X$ and each $\lambda \in Q$.

Note 2.4. When the field of scalars is $R$ or $C$ then the notion of left and right s.i.p. coincides with the notion of s.i.p. introduced by Lumer (1961) and Giles (1967).

We quote the following result which is straightforward from the generalised Hahn-Banach theorem. 
TheOREM 2.5. Let $X$ be a normed space over $Q$. For each arbitrary $x_{0} \in X$ $\left(x_{0} \neq 0\right)$ there exist functional $f_{l} \in X_{l}^{*}$ and $f_{r} \in X_{r}^{*}$ such that $f_{l}\left(x_{0}\right)=\left\|x_{0}\right\|,\left\|f_{l}\right\|=1$ and $f_{r}\left(x_{0}\right)=\left\|x_{0}\right\|,\left\|f_{r}\right\|=1$. Here $X_{l}^{*}\left(X_{r}^{*}\right)$ is the space of all bounded left (right) linear functionals on $X$.

It can be easily verified that every left and right s.i.p. space over $Q$ is a normed space over $Q$.

Lumer (1961) has shown that every normed space over $R$ or $C$ can be represented as a s.i.p. space (in general in infinitely many different ways); we now generalise this result as follows.

THEOREM 2.6. Every normed space over $Q$ can be represented as a left s.i.p. space over $Q$ (in general in infinitely many different ways).

Proof. Let us introduce on the unit sphere $S$ of $X$ an equivalence relation $\sim$ by $s_{1} \sim s_{2}$ if and only if there exist $\alpha_{1}, \alpha_{2} \in Q\left(\left|\alpha_{1}\right|=\left|\alpha_{2}\right|=1\right)$ such that $s_{1}=\alpha_{1} s_{2} \alpha_{2}$ ( $\alpha_{1}$ and $\alpha_{2}$ are unique). Let $S_{0}$ be any equivalence class. By the axiom of choice, we can select an element $s_{0} \in S_{0}$. Now by the generalised Hahn Banach theorem, there exists an $f_{S_{0}} \in X_{l}^{*}$ such that $f_{S_{0}}\left(s_{0}\right)=1$ and $\left\|f_{S_{0}}\right\|=1$. For each $s \in S_{0}$, $s=\alpha_{1} s_{0} \alpha_{2}\left(\left|\alpha_{1}\right|=\left|\alpha_{2}\right|=1\right)$. We define $f_{S}=f_{\alpha_{1} S_{0} \alpha_{2}}=\bar{\alpha}_{2} f_{S_{0}} \bar{\alpha}_{1}$. Since $f_{S_{0}}$ is a left linear functional then so also is $f_{S}$.

Now $f_{S}(s)=\left(\bar{\alpha}_{2} f_{S_{0}} \bar{\alpha}_{1}\right)\left(\alpha_{1} s_{0} \alpha_{2}\right)=\left(\bar{\alpha}_{2} f_{S_{0}}\right)\left(\alpha_{1} s_{0} \alpha_{2}\right) \bar{\alpha}_{1}=f_{S_{0}}\left(\alpha_{1} s_{0} \alpha_{2} \bar{\alpha}_{2}\right) \bar{\alpha}_{1}=1$. For arbitrary $y \in X(y \neq 0)$,

$$
y=\|y\| \cdot s=\|y\| \alpha_{1} s_{0} \alpha_{2} \quad\left(\left|\alpha_{1}\right|=\left|\alpha_{2}\right|=1, s_{0} \in S_{0}\right) .
$$

We define $f_{y}=\|y\| f_{S}=\|y\| \bar{\alpha}_{2} f_{S_{0}} \bar{\alpha}_{1}$ and $f_{0}=0$. Now let $\beta \in Q$ and $\beta=|\beta| \beta_{0}$ $\left(\left|\beta_{0}\right|=1\right)$. Then $f_{\beta y}=f_{|\beta||y| \mid \beta_{0} \alpha_{1} S_{0} \alpha_{2}}=|\beta| \cdot\|y\| \bar{\alpha}_{2} f_{S_{0}} \bar{\alpha}_{1} \bar{\beta}_{0}=f_{y} \bar{\beta}$.

Similarly, $f_{y \beta}=\bar{\beta} f_{y}$.

We define a transformation $W: X \rightarrow X_{l}^{*}, W(y)=f_{y}$. Then from above, this transformation has the following properties:

1) $f_{y}(y)=\|y\|^{2}(\forall y \in X)$,

2) $f_{\beta y}=f_{y} \bar{\beta}(\beta \in Q)$,

3) $f_{y \beta}=\bar{\beta} f_{y}(\beta \in Q)$,

4) $\left\|f_{y}\right\|=\|y\|$.

For each $x, y \in X$, we define $(x, y)=f_{y}(x)$. Then it can be easily verified that this defines a left s.i.p. on $X$. Hence $X$ is a left s.i.p. space over $Q$. In general there are many such mappings $W$ from $X$ to $X_{l}^{*}$. Therefore $X$ can be represented as a left s.i.p. space over $Q$ in infinitely many different ways.

By symmetry, the following result also holds. 
THEOREM 2.7. Every normed space over $Q$ can be represented as a right s.i.p. space over $Q$ (in general in infinitely many different ways).

NoTE 2.8. Torgashev (1974), in Theorem 1, has proved that every normed space over $Q$ can be represented as a left s.i.p. with properties $\left(b^{\prime}\right)$ and $\left(b^{\prime \prime}\right)$ of Definition 2.1.

Note 2.9. Let $X$ be a normed space over $Q$. Then $X$ is also a normed space over $R$, which will be denoted by $X_{R}$. If $f_{0}$ is any real linear functional on $X_{R}$ we define for $x \in X$,

$$
f_{l}(x)=f_{0}(x)-i f_{0}(i x)-j f_{0}(j x)-k f_{0}(k x)
$$

and

$$
f_{r}(x)=f_{0}(x)-i f_{0}(x i)-j f_{0}(x j)-k f_{0}(x k),
$$

Then $f_{l} \in X_{l}^{*}$ and $f_{r} \in X_{r}^{*}$.

Conversely, if $f_{l}$ and $f_{r}$ are elements of $X_{l}^{*}$ and $X_{r}^{*}$ respectively such that $\operatorname{Re}\left(f_{l}\right)=\operatorname{Re}\left(f_{r}\right)=f_{0}$ (say), then $f_{0}$ is a real linear functional on $X_{R}$, and further $f_{l}$ and $f_{r}$ satisfy (1) and (2). Now we define the map $T: X_{l}^{*} \rightarrow X_{r}^{*}$ by $T\left(f_{l}\right)=f_{r}$ where $f_{l}$ and $f_{r}$ are given by (1) and (2). Then it can be easily verified that $\left\|f_{l}\right\|=\left\|f_{0}\right\|=$ $\left\|T\left(f_{l}\right)\right\|=\left\|f_{r}\right\|$ and hence $T$ is a real linear isometry from $X_{l}^{*}$ onto $X_{r}^{*}$.

We now show that left and right s.i.p. products are equivalent in a certain sense.

THEOREM 2.10. Let (, ) be a left s.i.p. on a normed space $X$ over $Q$. Then a right s.i.p. $\langle$,$\rangle can be derived, and conversely.$

Proof. Let ( , ) be a left s.i.p. on $X$. Suppose $[x, y]=\operatorname{Re}(x, y)$ for all $x, y \in X$. Then $[$,$] is a real s.i.p. on X_{R}$ and $(x, y)=[x, y]-i[i x, y]-j[j x, y]-$ $k[k x, y]$. From this, $[x, x]=(x, x)=\|x\|^{2}$ and $[i x, x]=[j x, x]=[k x, x]=0$ for all $x \in X$.

Let $\lambda \in Q$ and $\lambda=a_{1}+i a_{2}+j a_{3}+k a_{4}$ say. Now

$$
[\lambda x, y]=a_{1}[x, y]+a_{2}[i x, y]+a_{3}[j x, y]+a_{4}[k x, y] .
$$

Again,

$$
[x, \lambda y]=\operatorname{Re}(x, \lambda y)=\operatorname{Re}\{(x, y) \bar{\lambda}\}=[\bar{\lambda} x, y]
$$


and

(iii)

$$
\begin{aligned}
{[x, y \lambda] } & =\operatorname{Re}(x, y \lambda)=\operatorname{Re}(x \bar{\lambda}, y) \\
& =a_{1}[x, y]-a_{2}[x i, y]-a_{3}[x j, y]-a_{4}[x k, y] .
\end{aligned}
$$

Let us define a functional $\langle$,$\rangle on X$ by

$$
\langle x, y\rangle=[x, y]-i[x i, y]-j[x j, y]-k[x k, y] \text {. }
$$

Then we have

(a) $\langle x+z, y\rangle=\langle x, y\rangle+\langle z, y\rangle$,

(b') $\langle x \lambda, y\rangle=[x \lambda, y]-i[x \lambda i, y]-j[x \lambda j, y]-k[x \lambda k, y]=\langle x, y\rangle \lambda$,

$\left(b^{\prime \prime}\right)\langle x, y \lambda\rangle=[x, y \lambda]-i[x i, y \lambda]-j[x j, y \lambda]-k[x k, y \lambda]=\bar{\lambda}\langle x, y\rangle$, by the relation (iii),

$\left(\mathrm{b}^{\prime \prime \prime}\right)\langle\lambda x, y\rangle=[\lambda x, y]-i[\lambda x i, y]-j[\lambda x j, y]-k[\lambda x k, y]=\langle x, \vec{\lambda} y\rangle$, by the relation (ii),

(c) $\langle x, x\rangle \geqslant 0$, $=0$ only if $x=0$,

(d) by Note 2.9 and the Cauchy-Schwarz inequality for the real s.i.p. [ , ], we have $|\langle x, y\rangle| \leqslant\|x\| \cdot\|y\|$.

Therefore $\langle$,$\rangle defines a right s.i.p. on X$. The converse statement is proved similarly.

Note 2.11. Though left and right s.i.p. products are equivalent in a certain sense, they are needed for studying the interconnection between left and right complex strictly convex, $Q$-complex strictly convex and quaternion strictly convex spaces.

Further, let us recall that Torgashev (1976) defined the numerical range of a right linear operator by using left linear functionals. But a left s.i.p. is nothing but a set of left linear functionals.

We now give a characterization of Hilbert spaces over $Q$.

THEOREM 2.12. Let $X$ be a Banach space over $Q$. If there exists a left s.i.p. on $X$ such that $(x, y)+(y, x)$ is real for all $x, y \in X$, then $X$ is a Hilbert space.

Proof. Suppose (, ) is a left s.i.p. on $X$ which satisfies the condition that, for all $x, y \in X$,

(i) $(x, y)+(y, x)$ is real. Now in (i), by replacing $x$ by $i x$ we have

(ii) $i(x, y)-(y, x) i$ is real. From the conditions (i) and (ii) we have $(x, y)=$ $(\overline{y, x})$. Thus $($,$) is an inner product on X$ in the sense of Torgashev (1974). Hence $X$ is a Hilbert space. 


\section{Strict convexity}

We now introduce the concept of strict convexity in normed spaces over $Q$.

Definition 3.1. Let $X$ be a normed space over $Q$. We say $X$ is left (right) strictly convex if and only if every non-zero continuous left (right) linear functional attains its maximum on the unit sphere at most at one point.

THEOREM 3.2. A left (right) linear functional attains its maximum on the unit sphere at a unique point if and only if it's real part attains its maximum on the unit sphere at a unique point.

Proof. The result follows from Note 2.9.

THEOREM 3.3. Let $T$ be the map defined in Note 2.9. Then a left linear functional $f_{l}$ attains its maximum on the unit sphere at a unique point if and only if $T\left(f_{l}\right)$ attains its maximum on the unit sphere at a unique point.

Proor. The result follows from Note 2.9 and Theorem 3.2.

NoTE 3.4. From Theorem 3.3 we conclude that left strict convexity implies right strict convexity and conversely. Hence we simply use the phrase strictly convex (in short, s.c.).

NOTE 3.5. Hilbert spaces over $Q$ are s.c. spaces.

Note 3.6. From Theorem 3.2 we conclude that $X$ is s.c. if and only if $X_{R}$ is s.c.

We now generalise Theorem 1 of Beesack, Hughes and Ortel (1979).

TheOREM 3.7. A normed space $X$ over $Q$ is s.c. if and only if whenever $x, y \in X$ with $x \neq y$ and $\|x\|=1=\|y\|$, we have $\|q x+(1-q) y\|<1$ for some $q \in Q$.

Proof. Suppose $X$ is s.c. Then $X_{R}$ is s.c. and the result holds with $q=\frac{1}{2}$.

Conversely suppose the given condition is satisfied. If $X$ is not s.c. then there exist $x, y$ and a bounded left linear functional $f_{l}$, such that $f_{l}(x)=f_{l}(y)=\|x\|=$ $\|y\|=1=\left\|f_{l}\right\|$. Hence for any $q \in Q$,

$$
\|q x+(1-q) y\| \geqslant\left\|f_{l}(q x+(1-q) y)\right\|=1
$$

and so the given condition fails. 
Similarly the following result holds.

TheOREM 3.8. A normed space $X$ over $Q$ is s.c. if and only if whenever $x, y \in X$ with $x \neq y$ and $\|x\|=1=\|y\|$, we have $\|x q+y(1-q)\|<1$ for some $q \in Q$.

Results of Köethe (1969) (Theorem 3), Torrance (1970), Gudder and Strawther (1976) and Sen (1981) are also true in this setting.

\section{4. $C$-strict convexity and $Q$-strict convexity}

We recall the following definitions from Istratescu (1979a).

Definition 4.1. Let $K$ be a convex subset of a normed space $X$ over $C$ and $x_{0} \in K$. The element $x_{0}$ is a complex extreme point of $K$ if and only if $\{x+z y$ : $|z| \leqslant 1, z \in C\} \subset K$ for $y \in X$, implies $y=0$.

Definition 4.2. A normed space $X$ over $C$ is called complex strictly convex (c.s.c.) if and only if each point of the unit sphere is a complex extreme point of the unit ball.

Istratescu (1979b), Theorem 2.7, gave a sufficient condition for a Banach space to be c.s.c. An easy application of Theorem 1 of Holub (1975) shows that it is in reality a sufficient condition for a space to be s.c.

We now give a sufficient condition for a normed space over $C$ to be c.s.c.

Definition 4.3. For every normalized pair $\left\{x_{1}, x_{2}\right\}$ of linearly independent elements of a normed space $X$ over $C$, let $V\left[x_{1}, x_{2}\right]$ be the complex vector space spanned by $x_{1}$ and $x_{2}$ and let $R\left[x_{1}, x_{2}\right]$ be the set of all those elements $y=a_{1} x_{1}+a_{2} x_{2} \in V\left[x_{1}, x_{2}\right]$ with $a_{1} a_{2}$ real.

THEOREM 4.4. Let $X$ be a normed space over $C$. If for every normalized pair $\left\{x_{1}, x_{2}\right\}$ of linearly independent elements in $X$, the points in $V\left[x_{1}, x_{2}\right]$ equidistant from $x_{1}$ and $x_{2}$ are in $R\left[x_{1}, x_{2}\right]$ then $X$ is c.s.c.

Proof. Suppose that $X$ is not a c.s.c. space. Then there exist an $x \in X,\|x\|=1$ and $a y \neq 0$ such that $\|x+z y\|=1$ for all $|z| \leqslant 1, z$ complex. Let $x_{1}=i x+y$, $x_{2}=x+y$. Then $\left\|x_{1}\right\|=\left\|x_{2}\right\|=1$. 
Suppose $u=i x_{1}+x_{2}$. Then $u \in V\left[x_{1}, x_{2}\right]$. Now $u-x_{1}=-i(x-y)$ and $u-x_{2}=-x+i y$. As $\left\|u-x_{1}\right\|=\left\|u-x_{2}\right\|=1, u$ is equidistant from $x_{1}$ and $x_{2}$. Since $u$ is not in $R\left[x_{1}, x_{2}\right]$, we have a contradiction.

Note 4.5. Let $X$ be a complex normed space. Then $X$ can be considered as a normed space over the reals. If $x_{1}, x_{2}$ is a normalized pair of linearly independent elements of $X$, then $V\left[x_{1}, x_{2}\right]=V_{R}\left[x_{1}, x_{2}, i x_{1}, i x_{2}\right]$, where $V_{R}\left[x_{1}, x_{2}, i x_{1}, i x_{2}\right]$ is the real linear span of $x_{1}, x_{2}, i x_{1}, i x_{2}$.

Thus $V\left[x_{1}, x_{2}\right]$ can be considered as a real subspace of $X$ and $R\left[x_{1}, x_{2}\right]$ is a subset of $V\left[x_{1}, x_{2}\right]$.

Therefore it is relevant to ask whether in Theorem 4.4, c.s.c. can be replaced by s.c. But the author has not been able to settle this problem. The author has however observed that the condition in Theorem 4.4 is not necessary for s.c. For let $X=l_{2}$ and $e_{1}$ and $e_{2}$ be two mutuallyorthogonal unit vectors of $X$. Then the vector $(1+i)\left(e_{1}+e_{2}\right) \in V\left[e_{1}, e_{2}\right]$ and is equidistant from $e_{1}$ and $e_{2}$ but not in $R\left[e_{1}, e_{2}\right]$.

We now define left and right complex extreme points on a normed space over $Q$.

Definition 4.6. A quaternion $a_{0}+i a_{1}+j a_{2}+k a_{3}$ is said to be $Q$-complex (complex) if and only if at least one (two) of $a_{1}, a_{2}, a_{3}$ is (are) zero.

Definition 4.7. Let $C$ be a convex set of a normed space $X$ over $Q$. An element $x_{0} \in C$ is said to be a left complex extreme point of $C$ if and only if $\left\{x_{0}+z y\right.$ : $|z| \leqslant 1, z$ complex $\} \subset C$ for $y$ in $X$, implies $y=0$.

Similarly a right complex extreme point can be defined in the same manner.

Definition 4.8. A normed space $X$ over $Q$ is said to be left (right) complex strictly convex if and only if every element of the unit sphere is a left (right) complex extreme point of the unit ball. Similarly, we can define left and right $Q$-complex strict convexity and quaternion strict convexity.

NoTE 4.9. It would be interesting to know whether the left and right concepts are equivalent.

EXAMPLE 4.10. Let $l_{1}$ be the set of all infinite sequences of quaternions $x=\left\{x_{i}\right.$ : $i=1,2, \ldots\}$ such that $\sum_{i=1}^{\infty}\left|x_{i}\right|<\infty$. Addition and left and right scalar multiplications on $l_{1}$ are defined as usual; $l_{1}$ forms a Banach space over $Q$ with the norm $\|x\|=\sum_{i=1}^{\infty}\left|x_{i}\right|$. The space $l_{1}$ is both a left and right c.s.c. space; this follows from Corollary 4.23 . 
Note 4.11. Henceforth we only consider left properties as the corresponding right ones can be discussed similarly.

NoTe 4.12. Every left c.s.c. space is a left $Q$-c.s.c. space and evry left $Q$-c.s.c. space is a left q.s.c. space.

NoTE 4.13. It would be interesting to find an example of a left $Q$-c.s.c. space which is not left c.s.c. and another example of a left q.s.c. space which is not a left $Q$-c.s.c. space.

We now give a sufficient condition for a normed space over $Q$ to be left c.s.c.

Definition 4.14. For every normalized pair $\left\{x_{1}, x_{2}\right\}$ of left linearly independent elements of a normed space $X$ over $Q$, let $V_{l}\left[x_{1}, x_{2}\right]$ be the left complex vector space spanned by $x_{1}, x_{2}$ and let $R_{l}\left[x_{1}, x_{2}\right]$ be the set of all $y=a_{1} x_{1}+$ $a_{2} x_{2} \in V_{l}\left[x_{1}, x_{2}\right]$ with $a_{1} \cdot a_{2}$ real.

THEOREM 4.15. Let $X$ be a normed space over $Q$. If for every normalized pair $\left\{x_{1}, x_{2}\right\}$ of left linearly independent elements in $X$, the points in $V_{l}\left[x_{1}, x_{2}\right]$ equidistant from $x_{1}$ and $x_{2}$ are in $R\left[x_{1}, x_{2}\right]$, then $X$ is a left c.s.c. space.

Proof. The proof is the same as for Theorem 4.4.

We now give a sufficient condition for a normed space over $Q$ to be left $Q$-c.s.c.

DEFINITION 4.16. For every normalized pair $\left\{x_{1}, x_{2}\right\}$ of left linearly independent elements of a normed space $X$ over $Q$, let $V_{l}\left[x_{1}, x_{2}\right]$ be the left $Q$-complex vector space spanned by $x_{1}, x_{2}$ and let $C_{l}\left[x_{1}, x_{2}\right]$ be the set of all $y=a_{1} x_{1}+$ $a_{2} x_{2} \in V_{l}\left[x_{1}, x_{2}\right]$ with $a_{1} \cdot a_{2}$ complex.

THEOREM 4.17. Let $X$ be a normed space over $Q$. If for every normalized pair $\left\{x_{1}, x_{2}\right\}$ of left linearly independent elements in $X$, the points in $V_{l}\left[x_{1}, x_{2}\right]$ equidistant from $x_{1}$ from $x_{2}$ are in $C_{l}\left[x_{1}, x_{2}\right]$, then $X$ is left $Q-c . s . c$.

Proof. Suppose that $X$ is not a left $Q$-c.s.c. space. Then there exist an $x \in X$, $\|x\|=1$, and $y \neq 0$ such that $\|x+q y\|=1$ for all $Q$-complex $q$ with $|q| \leqslant 1$. Let $x_{1}=x$ and $x_{2}=x-k y$. Then $\left\|x_{1}\right\|=\left\|x_{2}\right\|=1$.

Suppose $u=(i+j) x_{1}+x_{2}$. Then $u \in V_{l}\left[x_{1}, x_{2}\right]$. Now $u-x_{1}=(i+j-1) x$ $+x-k y=(i+j) x-k y$ and $u-x_{2}=(i+j) x$. 
From this, $\left\|u-x_{1}\right\|=|i+j| \cdot\left\|x-(i+j)^{-1} k y\right\|=\sqrt{2}$, as $(i+j)^{-1} k$ is $Q$-complex and $\left|(i+j)^{-1} k\right|<1$ and $\left\|u-x_{2}\right\|=\sqrt{2}$. Therefore $u$ is equidistant from $x_{1}$ and $x_{2}$. Since $u$ is not in $C_{l}\left[x_{1}, x_{2}\right]$, we have a contradiction.

We now give a sufficient condition for a normed space over $Q$ to be left q.s.c.

Definition 4.18. For every normalized pair $\left\{x_{1}, x_{2}\right\}$ of left linearly independent elements of a normed space $X$ over $Q$, let $V_{l}\left[x_{1}, x_{2}\right]$ be the left quaternion vector space spanned by $x_{1}, x_{2}$ and let $Q_{l}\left[x_{1}, x_{2}\right]$ be the set of all $y=a_{1} x_{1}+$ $a_{2} x_{2} \in V_{l}\left[x_{1}, x_{2}\right]$ with $a_{1} \cdot a_{2} Q$-complex.

THEOREM 4.19. Let $X$ be a normed space over $Q$. If for every normalized pair $\left\{x_{1}, x_{2}\right\}$ of left linearly independent elements in $X$, the points in $V_{l}\left[x_{1}, x_{2}\right]$ equidistant from $x_{1}$ and $x_{2}$ are in $Q_{l}\left[x_{1}, x_{2}\right]$, then $X$ is left q.s.c.

Proof. Suppose that $X$ is not a left q.s.c. space. Then there exist an $x \in X$, $\|x\|=1$, and a $y \neq 0$ such that $\|x+q y\|=1$ for all $|q| \leqslant 1, q$ quaternion. Let $x_{1}=x+y$ and $x_{2}=x-y$. Then $\left\|x_{1}\right\|=\left\|x_{2}\right\|=1$. Suppose $u=(i+1) x_{1}+$ $(j+i) x_{2}$. Then it can be easily verified that $u$ is equidistant from $x_{1}$ and $x_{2}$ but not in $Q_{l}\left[x_{1}, x_{2}\right]$. Hence we have a contradiction.

We now give a characterization of left q.s.c. product spaces with $l_{p}$-norm.

Before proving our required characterization we prove a lemma.

LeMma 4.20. Let $X$ be a normed space over $Q$. If $\|u\|=1$ and $\|u \pm v\|=1$ then $(v, u)=0=\langle v, u\rangle$, where $($,$) and \langle$,$\rangle denote left and right s.i. products on X$ respectively.

Proof. We have $|1 \pm(v, u)|=|(u \pm v, u)| \leqslant\|u \pm v\| \cdot\|u\|=1$ or $|1 \pm(v, u)|$ $\leqslant 1$. Since the space $Q$ of quaternions with usual norm is strictly convex this implies that $(v, u)=0$. Similarly $\langle v, u\rangle=0$.

THEOREM 4.21. Let $X$ be the product space $X_{1} \times X_{2}$ with $l_{p}$-norm $(1 \leqslant p \leqslant \infty)$. For $p=\infty, X$ is never left q.s.c. For $1 \leqslant p<\infty, X$ is left q.s.c. if and only if each $X_{i}(i=1 ; 2)$ is left q.s.c.

Proof. Let $X$ be left q.s.c. and $1 \leqslant p<\infty$. If the result does not hold then there exists an $X_{i}$ (say $X_{1}$ ) such that $X_{1}$ is not left q.s.c. Since $X_{1}$ is not left q.s.c. then there exist a $u,\|u\|=1$ and a $v \neq 0$ such that $\|u+q v\|=1$ for all $|q| \leqslant 1$. We choose $x=(u, 0), y=(v, 0)$. 
Then $\|x\|=1$ and $\|x+q y\|=1,|q| \leqslant 1$. Since $X$ is left q.s.c., $y=0$, a contradiction.

We now prove the converse.

Case I, $1<p<\infty$. Let each $X_{i}(i=1,2)$ be left q.s.c. Suppose [,$]_{X_{i}}$ is any consistent left s.i.p. on $X_{i}(i=1,2)$. Then a left s.i.p. on $X$ is given by

$$
\left[\left(x_{1}, x_{2}\right),\left(y_{1}, y_{2}\right)\right]=\frac{\Sigma_{i=1}^{2}\left[x_{i}, y_{i}\right]_{X_{i}} \cdot\left\|y_{i}\right\|^{p-2}}{\left\|\left(y_{1}, y_{2}\right)\right\|^{p^{-2}}}, \quad 1<p<\infty .
$$

Let $u, v \in X,\|u\|=1$ and $\|u+q v\|=1,|q| \leqslant 1$. Then by Lemma 4.20, $[v, u]=0$. If $u=\left(x_{1}, x_{2}\right), v=\left(y_{1}, y_{2}\right)$, then

$$
\begin{aligned}
1 & =\left\|x_{1}\right\|^{p}+\left\|x_{2}\right\|^{p}=\left[x_{1}+q y_{1}, x_{1}\right]_{X_{1}} \cdot\left\|x_{1}\right\|^{p-2}+\left[x_{2}+q y_{2}, x_{2}\right]_{X_{2}} \cdot\left\|x_{2}\right\|^{p-2} \\
& \leqslant\left\|x_{1}+q y_{1}\right\| \cdot\left\|x_{1}\right\|^{p-1}+\left\|x_{2}+q y_{2}\right\| \cdot\left\|x_{2}\right\|^{p-1} \\
& \leqslant\left(\left\|x_{1}+q y_{1}\right\|^{p}+\left\|x_{2}+q y_{2}\right\|^{p}\right)^{1 / p} \cdot\left(\left\|x_{1}\right\|^{(p-1) q}+\left\|x_{2}\right\|^{(p-1) q}\right)^{1 / q}=1,
\end{aligned}
$$

Therefore by Hölder's inequality (note that $1 / p+1 / q=1$ ).

(i) $\left\|x_{1}+q y_{1}\right\| \cdot\left\|x_{1}\right\|^{p-1}+\left\|x_{2}+q y_{2}\right\| \cdot\left\|x_{2}\right\|^{p-1}$

$$
=\left(\left\|x_{1}+q y_{1}\right\|^{p}+\left\|x_{2}+q y_{2}\right\|^{p}\right)^{1 / p} \cdot\left(\left\|x_{1}\right\|^{p}+\left\|x_{2}\right\|^{p}\right)^{1 / q}=1 .
$$

Since equality holds in Hölder's inequality there exists a non-zero positive number $K_{q}$ such that

$$
\left\|x_{1}+q y_{1}\right\|^{p}=K_{q} \cdot\left\|x_{1}\right\|^{p}, \quad\left\|x_{2}+q y_{2}\right\|^{p}=K_{q} \cdot\left\|x_{2}\right\|^{p}
$$

for $|q| \leqslant 1$.

Now from (i) and (ii), $K_{q}=1$. Again since each $X_{i}$ is left q.s.c. then from (ii), $y_{i}=0$ for $i=1,2$.

Hence $v=0$. Therefore $X$ is left q.s.c.

Case II, $p=1$. Suppose each $X_{i}(i=1,2)$ is left q.s.c. Let $u, v \in X,\|u\|=1$ and $\|u+q v\|=1$ for all $|q| \leqslant 1$. Suppose $u=\left(x_{1}, x_{2}\right), v=\left(y_{1}, y_{2}\right)$. Then for $|q| \leqslant 1$,

$$
\left\|x_{1}\right\|+\left\|x_{2}\right\|=1, \quad\left\|x_{1}+q y_{1}\right\|+\left\|x_{2}+q y_{2}\right\|=1 .
$$

Without loss of generality we may assume that $\left\|x_{1}\right\|,\left\|x_{2}\right\| \neq 0$. Let $G_{1}=\{q$ : $\left.|q| \leqslant 1,\left\|x_{1}+q y_{1}\right\| \leqslant\left\|x_{1}\right\|\right\}$ and $G_{2}=\left\{q:|q| \leqslant 1,\left\|x_{2}+q y_{2}\right\| \leqslant\left\|x_{2}\right\|\right\}$. Then $G_{1} \cup$ $G_{2}=B$, the closed unit ball of $Q$, and $G_{1}$ and $G_{2}$ are closed sets.

Suppose $I=G_{1} \cap G_{2}$. Then $0 \in I$.

We now show that $I$ contains more than one element. If possible let $I=\{0\}$. Then $B \backslash G_{1}=G_{2} \backslash\{0\}$ is an open set in $B$ and $B \backslash G_{2}=G_{1} \backslash\{0\}$ is an open set in $B$. 
Now $B \backslash\{0\}=\left(G_{1} \backslash\{0\}\right) \cup\left(G_{2} \backslash\{0\}\right)$, the union of two disjoint open sets, which is a contradiction (as $B_{1} \backslash\{0\}$ is a connected set). Therefore, $I$ contains more than one element.

We now show that $I$ is symmetric. Suppose $q \in I$. Then

$$
\left\|x_{1}+q y_{1}\right\| \leqslant\left\|x_{1}\right\|, \quad\left\|x_{2}+q y_{2}\right\| \leqslant\left\|x_{2}\right\| .
$$

Again,

$$
\begin{aligned}
2 \cdot\left\|x_{1}\right\| & \leqslant\left\|x_{1}+q y_{1}\right\|+\left\|x_{1}-q y_{1}\right\| \\
& \leqslant\left\|x_{1}\right\|+\left\|x_{1}-q y_{1}\right\|
\end{aligned}
$$

or

$$
\left\|x_{1}\right\| \leqslant\left\|x_{1}-q y_{1}\right\| .
$$

Similarly,

$$
\left\|x_{2}\right\| \leqslant\left\|x_{2}-q y_{2}\right\|
$$

From (i), (iii), and (iv)

$$
\left\|x_{1}\right\|+\left\|x_{2}\right\| \leqslant\left\|x_{1}-q y_{1}\right\|+\left\|x_{2}-q y_{2}\right\| \leqslant\left\|x_{1}\right\|+\left\|x_{2}\right\| .
$$

Therefore from (iii) and (iv)

$$
\left\|x_{1}-q y_{1}\right\|=\left\|x_{1}\right\|, \quad\left\|x_{2}-q y_{2}\right\|=\left\|x_{2}\right\|
$$

which shows that $-q \in I$.

We now show that $I=B$. Let $q \in I, q \neq 0$ and $q_{1} \in Q$ such that $\left|q_{1} \cdot q\right| \leqslant 1$. Then as $I$ is symmetric,

$$
\left\|x_{1} \pm q y_{1}\right\|=\left\|x_{1}\right\|, \quad\left\|x_{2} \pm q y_{2}\right\|=\left\|x_{2}\right\| .
$$

By our Lemma 4.20, $\left[y_{1}, x_{1}\right]_{X_{1}}=0=\left[y_{2}, x_{2}\right]_{X_{2}}$. Now

$$
\begin{aligned}
\left\|x_{1}\right\|^{2} & =\left[x_{1}+q_{1} \cdot q y, x_{1}\right]_{X_{1}} \\
& \leqslant\left\|x_{1}+q_{1} \cdot q y_{1}\right\| \cdot\left\|x_{1}\right\|
\end{aligned}
$$

or

(v)

$$
\left\|x_{1}\right\| \leqslant\left\|x_{1}+q_{1} \cdot q y_{1}\right\|
$$

Similarly,

(vi)

$$
\left\|x_{2}\right\| \leqslant\left\|x_{2}+q_{1} \cdot q y_{2}\right\|
$$

From (i), (v) and (vi),

$$
\begin{aligned}
\left\|x_{1}\right\|+\left\|x_{2}\right\| & \leqslant\left\|x_{1}+q_{1} \cdot q y_{1}\right\|+\left\|x_{2}+q_{1} \cdot q y_{2}\right\| \\
& \leqslant\left\|x_{1}\right\|+\left\|x_{2}\right\| .
\end{aligned}
$$

From this we conclude that $\left\|x_{1}+q_{1} q y_{1}\right\|=\left\|x_{1}\right\|$ and $\left\|x_{2}+q_{1} \cdot q y_{2}\right\|=\left\|x_{2}\right\|$. Hence $q_{1} \cdot q \in I$, and therefore $I=B$. 
Hence $\left\|x_{1}+q y_{1}\right\|=\left\|x_{1}\right\|$ and $\left\|x_{2}+q y_{2}\right\|=\left\|x_{2}\right\|$ for $q \in B$. Since $X_{1}, X_{2}$ are left q.s.c., $y_{1}=0=y_{2}$. Hence $v=0$. Therefore, $X$ is left q.s.c.

For $p=\infty$, let $u=\left(x_{1}, 0\right), v=\left(0, y_{2}\right)$ with $\left\|x_{1}\right\|=1=\left\|y_{2}\right\|$. Then $\|u+q v\|_{\infty}$ $=1$ for $|q| \leqslant 1$. Therefore, $X$ is not left q.s.c.

Corollary 4.22. If $X$ is an $l_{p}$-sum $(1 \leqslant p<\infty)$ of a sequence of normed spaces $\left\{X_{i}\right\}$ then $X$ is left q.s.c. if and only if each $X_{i}$ is left q.s.c.

Proof. Case I, $1<p<\infty$. The proof follows from Theorem 4.21 above.

Case II, $p=1$. Suppose each $X_{i}$ is left q.s.c. Let $\|x\|=1,\|x+q y\|=1$ for $q \in B, x, y \in X$. Suppose $x=\left(x_{1}, x_{2}, x_{3}, \ldots\right), y=\left(y_{1}, y_{2}, y_{3}, \ldots\right)$. Then $\sum_{i=1}^{\infty}\left\|x_{i}\right\|=1$ and $\sum_{i=1}^{\infty}\left\|x_{i}+q y_{i}\right\|=1=\sum_{i=1}^{\infty}\left\|x_{i}\right\|$ for $q \in B$.

Let $G_{i}=\left\{q: q \in B,\left\|x_{i}+q y_{i}\right\| \leqslant x_{i} \|\right\}$ and $\bar{G}_{2}=\{q: q \in B,\|\bar{x}+q \bar{y}\| \leqslant\|\bar{x}\|\}$, where $\bar{x}=\left(0, x_{2}, x_{3}, \ldots\right), \bar{y}=\left(0, y_{2}, y_{3} \ldots\right)$. Then $G \cup \bar{G}_{2}=B$ and each $G_{i}$ and $\bar{G}_{2}$ are closed sets.

Arguing as in the case of two spaces, we have $I=G_{1} \cap \bar{G}_{2}$ contains more than one element. Also arguing as in the case of two spaces we can show that

$$
\left\|x_{1}+q y_{1}\right\|=\left\|x_{1}\right\| \text { and }\|\bar{x}+q \bar{y}\|=\|\bar{x}\| \text { for } q \in B .
$$

Therefore $G_{1}=B$ and hence $y_{1}=0$ as $X_{1}$ is left q.s.c. Arguing in the same manner we get $G_{i}=B$ for all $i$ and hence $y_{i}=0$ for all $i$ as the $X_{i}$ 's are left q.s.c. Therefore $y=0$ and hence $X$ is left q.s.c.

COROLlaRY 4.23. Theorem 4.21 and Corollary 4.22 also hold for left Q-c.s.c. and c.s.c. spaces over $Q$ and c.s.c. spaces over C.

NoTE 4.24. For $p=2$, Theorem 4.21 above is proved for c.s.c. spaces over $C$ in Istratescu (1979a).

COROLlaRY 4.25. Let $X$ be the product space $X_{1} \times X_{2}$ with $l_{p}$ norm, $1 \leqslant p \leqslant \infty$. For $1<p<\infty, X$ is s.c. if and only if $X_{i}(i=1,2)$ is s.c. For $p=1$ or $p=\infty, X$ is never s.c.

COROLLARY 4.26. If $X$ is an $l_{p}$-sum $(1<p<\infty)$ of a sequence of normed spaces $\left\{X_{i}\right\}$ then $X$ is s.c. if and only if each $X_{i}$ is s.c.

We recall the following definition from Jamison (1982). 
Definition 4.27. Let $X$ be a complex Banach space and $f$ an analytic function defined on a domain $D$ with values in $X$. The space $X$ is said to have the strong maximum modulus property (SMMP) if and only if either $\|f(z)\|$ has no maximum on $D$ or $f(z)$ is constant on $D$.

THEOREM 4.28. If $X$ is an $l_{p}$-sum $(1 \leqslant p<\infty)$ of a sequence of complex Banach spaces $\left\{X_{i}\right\}$ then $X$ has SMMP if and only if each $X_{i}$ has $S M M P$.

Proof. The result follows from Corollary 4.23 above and Theorem 3.1 of Throp and Whitley (1967).

Note 4.29. For $p=1$, Theorem 4.28 above is stated without proof in Jamison (1982).

\section{Acknowledgement}

The author wishes to express his heart-felt thanks to Dr. T. K. Mukherjee of the Department of Mathematics, Jadavpur University, for his kind help in the preparation of this paper and to the referee for his valuable suggestions.

\section{References}

P. R. Beesack, E. Hughes and M. Ortel (1979), 'Rotund complex normed linear spaces', Proc. Amer. Math. Soc. 75, 42-44.

J. R. Giles (1967), 'Classes of semi inner product spaces', Trans. Amer. Math. Soc. 129, 436-446.

S. Gudder and D. Strawther (1976), 'Strictly convex normed linear spaces', Proc. Amer. Math. Soc. 59. 263-267.

J. R. Holub (1975), 'Rotundity, orthogonality and characterizations of inner product spaces', Bull. Amer. Math. Soc. 81 (6), 1087-1089.

V. I. Istratescu (1979a), 'On complex strictly convex spaces-I', J. Math. Anal. Appl. 70, 423-429.

V. I. Istratescu (1979b), 'On complex strictly convex spaces-II', J. Math. Anal. Appl. 71, 580-589.

J. E. Jamison (1982), 'Banach spaces with the strong maximum modulus property', Abstracts Amer. Math. Soc. 3, 355.

G. Köethe (1969), Topological vector spaces, Volume I (Springer-Verlag, Berlin).

G. Lumer (1961), 'Semi inner product spaces', Trans. Amer. Math. Soc. 100, $29-43$.

D. K. Sen (1981), 'Characterization of strict convexity', Bull. Calcutta Math. Soc. 73, 93-97.

A. Torgashev (1974), 'A generalisation of inner and semi inner product spaces', Math. Vesnik, 11(26), $301-311$.

A. Torgashev (1976), 'On the numerical range of an operator in a normed space', Math. Vesnik. 13(28), $118-124$. 
F. Torrance (1970), 'Strictly convex spaces via semi inner product space orthogonality', Proc. Amer. Math. Soc. 26, 108-110.

E. Throp and R. Whitley (1967), 'The strong maximum modulus theorem for analytic functions into a Banach space', Proc. Amer. Math. Soc. 18, 640-646.

Department of Pure Mathematics

University of Calcutta

35, Ballygunge Circular Road

Calcutta - 700019

India 